\title{
12th International Vilnius Conference on Probability Theory and Mathematical Statistics and 2018 IMS Annual Meeting on Probability and Statistics
}

Remigijus Leipus (Vilnius University, Lithuania)

The 12th Vilnius Conference on Probability Theory and Mathematical Statistics and the 2018 IMS Annual Meeting took place in Vilnius, one of the most beautiful cities in the Old Continent and capital of Lithuania, on 2-6 July 2018. The time of the conference is linked to an important date in the history of Lithuania - on 16 February 1918, the Act of Independence declared an independent State of Lithuania.

The Vilnius Conference on Probability and Mathematical Statistics has a long and successful history. The first Vilnius Conference on Probability Theory and Mathematical Statistics was organised in June 1973, becoming the first big international meeting on probability theory and mathematical statistics in the former Soviet Union. Now the conference has established itself as a must-attend, international, quadrennial event for many researchers in the field.

Many prominent probabilists and statisticians attended the conference. There were more than 180 invited talks. The Wald Lectures were delivered by Luc Devroye, the 2018 Le Cam Lecturer was Ruth Williams, the Ney-

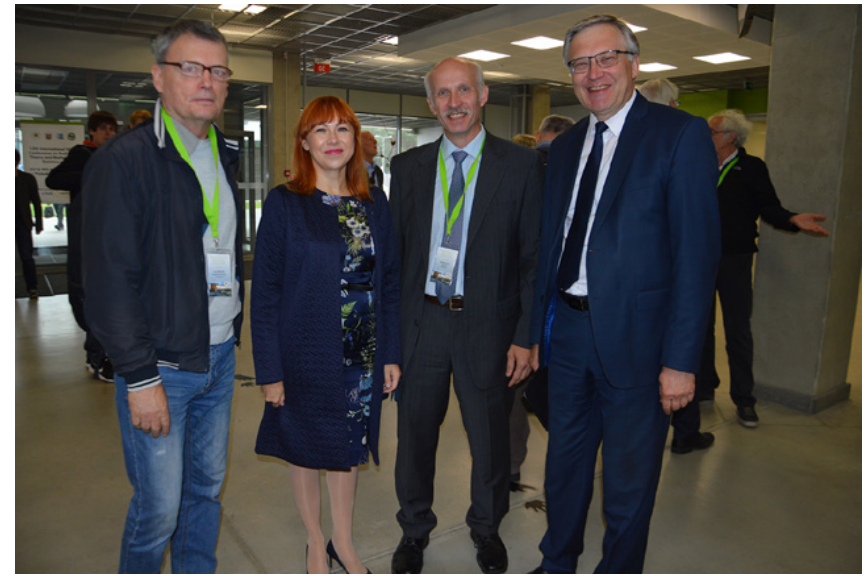

Alfredas Račkauskas, the Minister of Science and Education of Lithuania Jurgita Petrauskiené, Remigijus Leipus and the Rector of Vilnius University Artūras Žukauskas at the opening ceremony.

man Lecturer was Peter Bühlmann and the Schramm Lecturer was Yuval Peres. Six Medallion Lectures were given at this meeting, by Jean Bertoin, Svante Janson, 


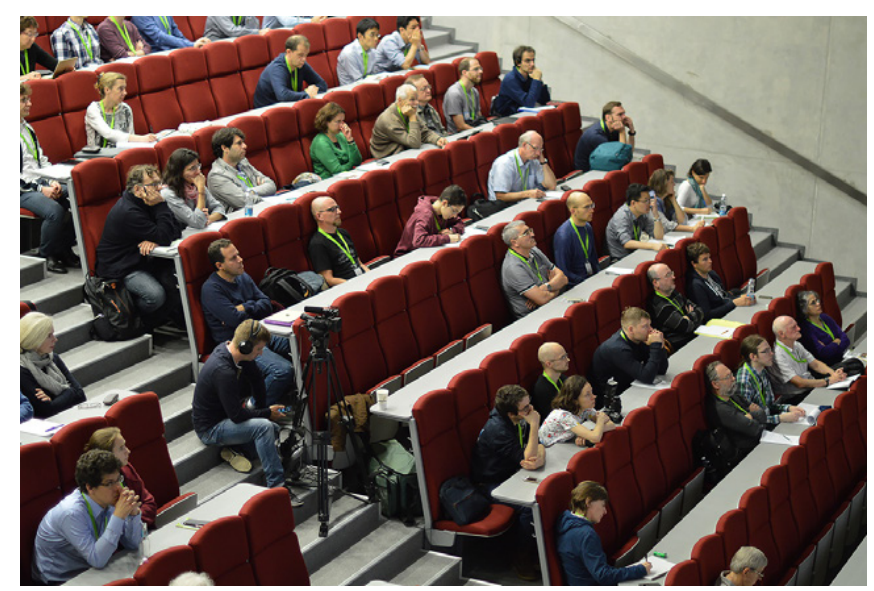

General view of the conference.

Thomas Mikosch, Sonia Petrone, Richard Samworth and Allan Sly. The Vilnius Lecture was delivered by Lithuanian probabilist Liudas Giraitis.

The EMS co-sponsored this event, supporting young scientists from developing countries. The EMS also established the EMS Gordin Prize to honour the memory of Mikhail Gordin. It was awarded to Mateusz Kwaśnicki (Wrocław University of Science and Technology) for his outstanding contributions to the spectral analysis of Lévy processes.

The organisers of the conference were the Lithuanian Mathematical Society, Vilnius University and the IMS. The programme co-chairs were Peter Bühlmann (IMS) and Vygantas Paulauskas (Vilnius). The organising committee co-chairs were Erwin Bolthausen (IMS) and Remigijus Leipus (Vilnius). The homepage of the conference is at http://ims-vilnius2018.com.

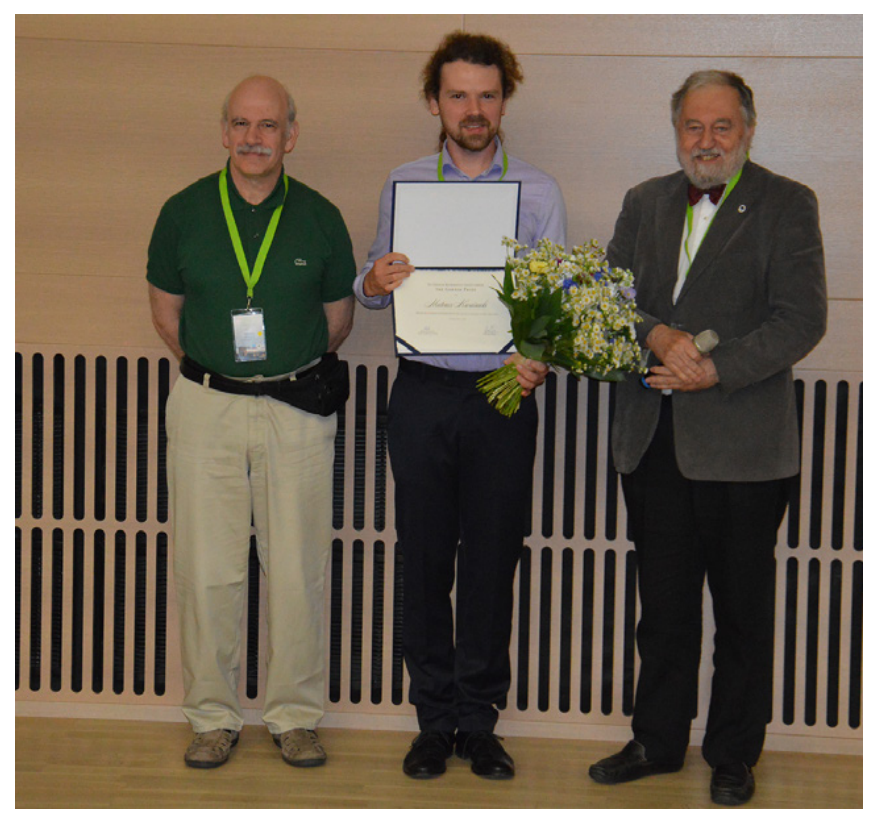

Mikhail Lifshits, Mateusz Kwaśnicki and Pavel Exner after the 2018 EMS Gordin Prize Award.

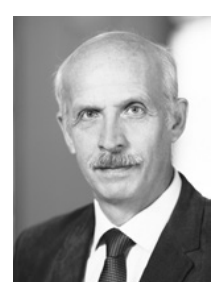

Remigijus Leipus is professor of Statistics and Probability at the Institute of Applied Mathematics, Vilnius University, Lithuania.His research interests are in probability theory, statistics, time series, insurance and financial mathematics, financial econometrics. At present he is the President of the Lithuanian Mathematical Society. 